\title{
Article \\ Dietary Intervention Induced Distinct Repercussions in Response to the Individual Gut Microbiota as Demonstrated by the In Vitro Fecal Fermentation of Beef
}

\author{
Vineet Singh, Youn-Chul Ryu and Tatsuya Unno *D
}

Citation: Singh, V.; Ryu, Y.-C.; Unno, T. Dietary Intervention Induced Distinct Repercussions in Response to the Individual Gut Microbiota as Demonstrated by the In Vitro Fecal Fermentation of Beef. Appl. Sci. 2021, 11, 6841. https://doi.org/10.3390/ app11156841

Academic Editor: Antonio Valero

Received: 24 June 2021

Accepted: 23 July 2021

Published: 25 July 2021

Publisher's Note: MDPI stays neutral with regard to jurisdictional claims in published maps and institutional affiliations.

Copyright: (c) 2021 by the authors. Licensee MDPI, Basel, Switzerland. This article is an open access article distributed under the terms and conditions of the Creative Commons Attribution (CC BY) license (https:// creativecommons.org/licenses/by/ $4.0 /)$.
Faculty of Biotechnology, School of Life Sciences, SARI, Jeju National University, Jeju 63243, Korea; vineet@jejunu.ac.kr (V.S.); ycryu@jejunu.ac.kr (Y.-C.R.)

* Correspondence: tatsu@jejunu.ac.kr; Tel.: +82-64-754-3354

\begin{abstract}
Animals and humans have very different gut microbiota, and the human microbiota is unique to each individual. For these reasons, it is difficult to find a diet that provides all the nutrients according to individual requirements. In this study, we investigated the possibility of using simple in vitro fecal fermentation of digested food to evaluate fundamental differences in the gut metabolism of individuals with different microbiomes in response to specific dietary interventions. We fermented beef using six human fecal microbiotas, analyzed shifts in these microbiomes, and quantified short-chain fatty acid (SCFA) production in each system. Our results demonstrate that each microbiome responds with a unique shift in composition, SCFA production, and metabolic activity following $90 \mathrm{~min}$ of fecal fermentation of beef. Differentially abundant genera and metabolic activities varied among subjects. Only two subjects' fecal microbiome showed no significant changes in their metabolic activity, while the other subjects' microbial metagenome showed anywhere between 17 and 60 differences in their metabolism, including several changes associated with heart disease (i.e., depletion of oleate and palmitoleate biosynthesis). This study revealed the varying responses of each microbiome when exposed to digested beef, suggesting that this method could provide fundamental information in understanding personal nutrient requirements and the impact of changes in the individual gut microbiota on human health. Although further studies using larger study populations are required, this study describes a simple and cost-effective protocol for evaluating the interactions between specific dietary interventions and individual gut microbiota differences.
\end{abstract}

Keywords: fecal fermentation; beef; fecal microbiome; dietary intervention; personal nutrient requirement

\section{Introduction}

The human gut harbors around $10^{14}$ microbes, comprising a genome size almost 150 times larger than that of the host itself [1,2]. These microbes interact with each other through various metabolites, such as short-chain fatty acids (SCFAs) and amines, to create a microenvironment inside the gut, which also affects the host. Studies have shown that gut microbes act differently in response to different diets, such as vegan, vegetarian, high-fat diet, Mediterranean, etc., and such dietary interventions have been reported to induce distinct changes in the microbial ecology and metabolic profile of the host $[3,4]$.

In the last decade, next-generation sequencing (NGS)-based culture-independent fecal microbiome analysis has been successfully used to analyze the effects of dietary interventions on the intestinal microbiome. The roles of individual dietary components, such as carbohydrates, proteins, and fats, have all been evaluated in an effort to understand their contribution to microbial diversity and population. Previous studies have shown that indigestible dietary fiber and polysaccharides are used by the gut microbiota to produce SCFAs. Three major SCFAs, acetic acid, propionic acid, and butyric acid, are known to play important roles in maintaining gut health. Similarly, amino acids produced by the 
enzymatic digestion of proteins can be used by gut microbes as an energy source and facilitate SCFA production [5]. Although amino acids are neither efficient energy sources nor preferentially consumed by gut microbes when compared to carbohydrates, studies have shown that amino acid fermentation results in highly diverse by-products, which can interact with the gut microbiome in many ways $[5,6]$. Previous studies have also shown that a protein diet can be metabolized by the gut microbiota to produce beneficial substances such as SCFAs and toxic substances, such as sulfur metabolites, depending on the type of microbes found in the gut [5-7].

Most evaluations of dietary interventions have relied on animal model data. However, there is a significant difference between the animal and the human gut microbiome, and each human microbiome is unique to the individual; thus, the effects of dietary interventions may vary depending on the composition of the gut microbiota in each individual. It would be ideal to use personal gut microbiota to investigate the effects of specific dietary interventions in each person, but the practicality of this approach remains lacking. The INFOGEST network was created by the European Cooperation in Science and Technology to standardize static in vitro digestion practices [8,9], which are harmonized according to the human body and adopted to mimic gastrointestinal digestion. This study used six unique fecal microbiota samples from six subjects to analyze the response of the fecal microbiome to the digestion of beef proteins using the INFOGEST protocol. Here, we investigated the possibility of using simple in vitro fecal fermentation to evaluate the fundamental differences in the metabolism of individual gut microbial communities in response to specific dietary interventions.

\section{Materials and Methods}

\subsection{In Vitro Gastrointestinal Digestion and Fecal Fermentation}

The frozen beef used in these experiments was obtained from the food market and stored at $-80^{\circ} \mathrm{C}$. All chemicals and enzymes used in these experiments were of analytical grade and were purchased from Sigma-Aldrich (St. Louis, MO, USA). All of the procedures in this study which involved human participants were conducted in accordance with the institutional ethical standards of Jeju National University, Korea (IRB approval number JJNU-IRB-2018-040-001) and the Helsinki declaration of 1964 and its later amendments or comparable ethical standards. Fecal samples were collected from six healthy adult donors (S1-6) and used to perform fecal fermentation. Donors were not on any medication for at least four weeks prior to taking part in the study.

The frozen meat was thawed and then minced to $2-3 \mathrm{~mm}[10,11]$ before heating to $60{ }^{\circ} \mathrm{C}$ for $2 \mathrm{~min}$ to complete the cooking process. The cooked meat was then used at $0.1 \% \mathrm{w} / \mathrm{v}$ to prepare $100 \mathrm{~mL}$ of fermentation broth, before gastrointestinal digestion was performed according to the established INFOGEST method [9]. The cooked meat was incubated in $5 \mathrm{~mL}$ phosphate-buffered saline (PBS) at $37^{\circ} \mathrm{C}$ for $2 \mathrm{~min}$ to normalize the temperature and then mixed with $3.5 \mathrm{~mL}$ of simulated salivary fluid $\left(1500 \mathrm{U} \cdot \mathrm{mL}^{-1} \alpha\right.$ - amylase $(0.5 \mathrm{~mL})$, $25 \mu \mathrm{L}$ of $\left.0.3 \mathrm{~mol} \cdot \mathrm{L}^{-1} \mathrm{CaCl}_{2}\right)$ ) and the volume was made up to $10 \mathrm{~mL}$ using distilled water. The enzymatic contact time was $2 \mathrm{~min}$ at $37^{\circ} \mathrm{C}$ with constant shaking at $100 \mathrm{rpm}$. For simulated gastric digestion, the prepared bolus $(10 \mathrm{~mL})$ was then mixed with $7.5 \mathrm{~mL}$ of simulated gastric fluid $\left(1.6 \mathrm{~mL}\right.$ of $25,000 \mathrm{U} \cdot \mathrm{mL}^{-1}$ pepsin, $5 \mu \mathrm{L}$ of $\left.0.3 \mathrm{~mol} \cdot \mathrm{L}^{-1} \mathrm{CaCl}_{2}\right)$ and $200 \mu \mathrm{L}$ of $4 \mathrm{~mol} \cdot \mathrm{L}^{-1} \mathrm{HCl}$ to adjust the $\mathrm{pH}$ to between 2.5 and 3. Distilled water was added to a final volume of $20 \mathrm{~mL}$. Gastric digestion was then simulated for $2 \mathrm{~h}$, with constant shaking at $100 \mathrm{rpm}$. Then, to simulate intestinal digestion, the gastric chyme $(20 \mathrm{~mL})$ was mixed with $11 \mathrm{~mL}$ of simulated intestinal fluid $\left(5 \mathrm{~mL}\right.$ of $800 \mathrm{U} \cdot \mathrm{mL}^{-1}$ pancreatin, $2.5 \mathrm{~mL}$ of $0.160 \mathrm{~mol} \cdot \mathrm{L}^{-1}$ bile salt, $40 \mu \mathrm{L}$ of $0.3 \mathrm{~mol} \cdot \mathrm{L}^{-1} \mathrm{CaCl}_{2}$ ) and $150 \mu \mathrm{L}$ of $1 \mathrm{~mol} \cdot \mathrm{L}^{-1} \mathrm{NaOH}$ to normalize the $\mathrm{pH}$ to 7 . This mixture was then made up to a final volume of $40 \mathrm{~mL}$ using distilled water. Intestinal digestion was performed for $2 \mathrm{~h}$ at $100 \mathrm{rpm}$ to thoroughly mix the components. The digested fluid was rapidly frozen in liquid nitrogen to stop the enzymatic reaction and then stored at $-20^{\circ} \mathrm{C}$. Further, digested fluid was freeze-dried to obtain a dried beef powder to be used in fecal fermentation. 
Basal fecal fermentation medium was prepared in triplicate as previously described [12], with one minor modification: both the peptones and yeast extracts were excluded from this broth as they may serve as alternate carbon sources. The basal fermentation media and the PBS buffers were placed in an anaerobic chamber for one day to remove any oxygen from their composition. Fresh feces were collected from six adult healthy male donors of age $27-45$ years, mixed with PBS (10\% $w / v)$, and then sieved using $250 \mu \mathrm{m}, 150 \mu \mathrm{m}$, and $25 \mu \mathrm{m}$ pore size filters. The extracted fecal slurries were then immediately transferred into an anaerobic chamber (Bactron II, SHEL LAB, Cornelius, North Carolina, USA) and inoculated into the prepared medium $(10 \% v / v)$. Digested beef powder was then added to the beef treatment group, and inoculated mixtures were incubated at $37^{\circ} \mathrm{C}$ and $100 \mathrm{rpm}$ for $90 \mathrm{~min}$, during which samples were collected at four time points $(0,30,60$ and $90 \mathrm{~min})$ along with their respective control (B0, B30, B60 and B90), where the content of controls was the same as beef-treated samples, except they were did not receive beef. After commencement of fecal fermentation, samples were immediately frozen using liquid nitrogen and stored at $-80{ }^{\circ} \mathrm{C}$ for further analysis. Later, on the basis of GC results, a suitable duration of fecal fermentation was determined to conduct the microbiome study.

\subsection{Quantitative Analysis of SCFAs}

Frozen fermented samples were thawed on ice and then $200 \mu \mathrm{L}$ of each sample was added to $1 \mathrm{~mL}$ of absolute methanol $(1: 5, v / v)$ and homogenized for 2 min using a vortex. Then, $4 \mathrm{M} \mathrm{HCI}$ was used to adjust the $\mathrm{pH}$ to 2. Samples were incubated for $10 \mathrm{~min}$ at room temperature with repeated vortexing every $2 \mathrm{~min}$, and then centrifuged at 10,000 rpm at $4{ }^{\circ} \mathrm{C}$ for $1 \mathrm{~min}$. The supernatant was collected using a $1 \mathrm{~mL}$ syringe, filtered through a $0.45 \mu \mathrm{m}$ membrane into a new sterile tube, and then stored frozen in liquid nitrogen. The quantitative analysis of the three main SCFAs (acetate, propionate and butyrate) was performed by gas chromatography (GC) (QP2010, Shimadzu, Japan) in split-less mode using a DB-FRAP column $(30 \mathrm{~m} \times 0.25 \mu \mathrm{m} \times 0.25 \mu \mathrm{m}$, Agilent, Santa Clara, CA, USA). The samples were injected onto the column maintained at $60{ }^{\circ} \mathrm{C}$. After $3 \mathrm{~min}$, the oven temperature was gradually increased to $200{ }^{\circ} \mathrm{C}$ at a rate of $20^{\circ} \mathrm{C} \cdot \mathrm{min}^{-1}$, held for $0.5 \mathrm{~min}$, and then increased to $230^{\circ} \mathrm{C}$ at a rate of $20^{\circ} \mathrm{C} \cdot \mathrm{min}^{-1}$ and maintained for $1.5 \mathrm{~min}$.

\subsection{DNA Extraction and $16 S$ rRNA Gene Sequencing}

Bacterial community analysis was completed using DNA extracted from the fermented samples, i.e., beef-treated samples along with their control samples. All frozen samples were thawed on ice, and $1 \mathrm{~mL}$ of sample was centrifuged at $10,000 \mathrm{rpm}$ for $5 \mathrm{~min}$ at $4{ }^{\circ} \mathrm{C}$. The supernatant was discarded, and the bacterial DNA was extracted from the pellet using a QIAamp PowerFecal Pro DNA Kit (QIAGEN, Maryland, USA). DNA was concentrated to $5 \mathrm{ng} \cdot \mu \mathrm{L}^{-1}$ and two-step PCR targeting the V4 region of the $16 \mathrm{~S}$ rRNA gene was performed using forward primer (5'-TCG TCG GCA GCG TCA GAT GTG TAT AAG AGA CAG GTG CCA GCM GCC GCG GTA A-3') and reverse primer (5'-GTC TCG TGG GCT CGG AGA TGT GTA TAA GAG ACA GGG A CT ACH VGG GTW TCT AAT-3'). An Illumina MiSeq (Illumina, San Diego, USA) library was then prepared according to the manufacturer's instructions and sent to Lab Genomics Inc. (Seongnam, South Korea) for sequencing.

\subsection{Fecal Microbiome Data Analysis}

The 16S rRNA gene sequencing data were analyzed using the mothur pipeline [13]. Briefly, paired-reads were merged with make.contigs and reads shorter than 200 bp or longer than 400 bp were removed by screen.seqs before being aligned to the SILVA database [14] using align.seqs. Machinery sequencing errors were corrected using pre.cluster, chimeric sequences were detected using chimera.vserach, and taxonomic classification was performed using the RDP database [15]. $\alpha$-diversity indices for species richness (Chao I) and species evenness (Shannon) were calculated using summary.single, and $\beta$-diversity was compared based on the Bray-Curtis distance [16], which was expressed using non-metric multidimen- 
sional scaling (NMDS). Intestinal metabolic activities were predicted using phylogenetic investigation of communities by reconstruction of unobserved states (PICRUSt2) [17].

\subsection{Statistical Analysis and Visualization}

Statistical differences in the SCFA and $\alpha$-diversity indices were estimated using analysis of variance (ANOVA). Microbial community differences were estimated using analysis of molecular variance (AMOVA). The differential abundance of microbes between the two groups was evaluated using linear discriminant analysis effect size (LEfSe) and linear discriminant analysis (LDA) in which scores higher than 3.0 and $p$-values of less than 0.05 were considered significant [18]. Microbial community types for each fecal donor were identified based on the Dirichlet multinomial mixtures as identified by the get.communitytype mothur subroutine using default parameters [19]. STAMP [20] was used to perform the Wilcoxon t-test to evaluate significant differences in metabolic activities with adjusted $p$ values by Bonferroni multiple testing correction. A heatmap was drawn using R packages heatmap. 2 and pheatmap.

\section{Results}

\subsection{Diversity in the Fecal Microbiota of Different Fecal Donors}

Microbial community type analysis showed that all fecal donors presented with unique microbial communities (Table S1).

Alpha-diversity analysis showed that subject 4 had significantly higher species richness than the other subjects, while subject 6 had significantly lower species richness $(p<0.05)$ (Figure 1A). Species evenness comparisons showed that subject 6 had significantly lower species evenness than the others $(p<0.05)$ (Figure 1B). The microbial composition at the phylum level was also shown to exhibit clear separation between individuals. Subject 2 had the highest number of Firmicutes and subject 4 exhibited an increase in the number of Verrucomicrobia and Euryachaeota (Figure 1C) compared to the other samples. The inter-individual differences were more evident at the genus level and subject 6 was the most distinctively different sample, presenting with more Lactobacillus and less Prevotella (Figure 1D) than the other subjects. Subject 1 had more Faecalibacterium, subject 2 more Megamonas, subject 3 more Bifidobacterium and Prevotella, subject 4 more Methanobrevibacter and Akkermansia, and subject 5 had more Alloprevotella, Streptococcus, and Megasphaera (Figure 1D). Among the six subjects, subjects 1 and 3 showed similar microbial communities, while subjects 5 and 6 presented with distinctively different microbiomes when evaluated at the genus level.

\subsection{Effects of In Vitro Condition on Fecal Microbiota}

A higher amount of SCFAs was observed after $90 \mathrm{~min}$ of fecal fermentation with beef compared to other time points (30 or $60 \mathrm{~min}$ ) (Figure S2); thus, only $90 \mathrm{~min}$ samples were used for the microbiome analysis. In this study, we performed fecal fermentation for $90 \mathrm{~min}$, and evaluation revealed that there were no significant changes in the microbial community composition of $\mathrm{B} 0$ and $\mathrm{B} 90$, associated with this treatment protocol (AMOVA, $p>0.05$ ). However, differential abundance analysis revealed significant increases and decreases in specific genera, which varied between subjects. On average, three to four genera were either increased or decreased in each subject during the incubation period (Figure S1). Differential abundance analysis revealed that Escherichia/Shigella increased among three subjects, while Faecalibacterium, Ruminococcus, and Prevotella decreased in most of the study subjects.

\subsection{Effect of Digested Beef on SCFA Content and the Fecal Microbiome}

After adding digested beef to the fecal fermentation systems, we observed a gradual increase in the amount of SCFA produced over a $90 \mathrm{~min}$ period, while samples without the digested beef did not show the same increase in SCFAs (Figure S2). 
We observed a significant increase in acetic acid in all of the samples following the addition of digested beef powders to the fermentation broth $(p<0.05)$, while propionic acid did not increase in subject 4 and butyric acid did not increase in subjects 3,4 , and 6 (Figure 2). The increase in acetic acid was the highest, while the increase in butyric acid was the lowest. In addition, these evaluations revealed that the total concentration of SCFAs increased in half of the subjects in response to digested beef powder.
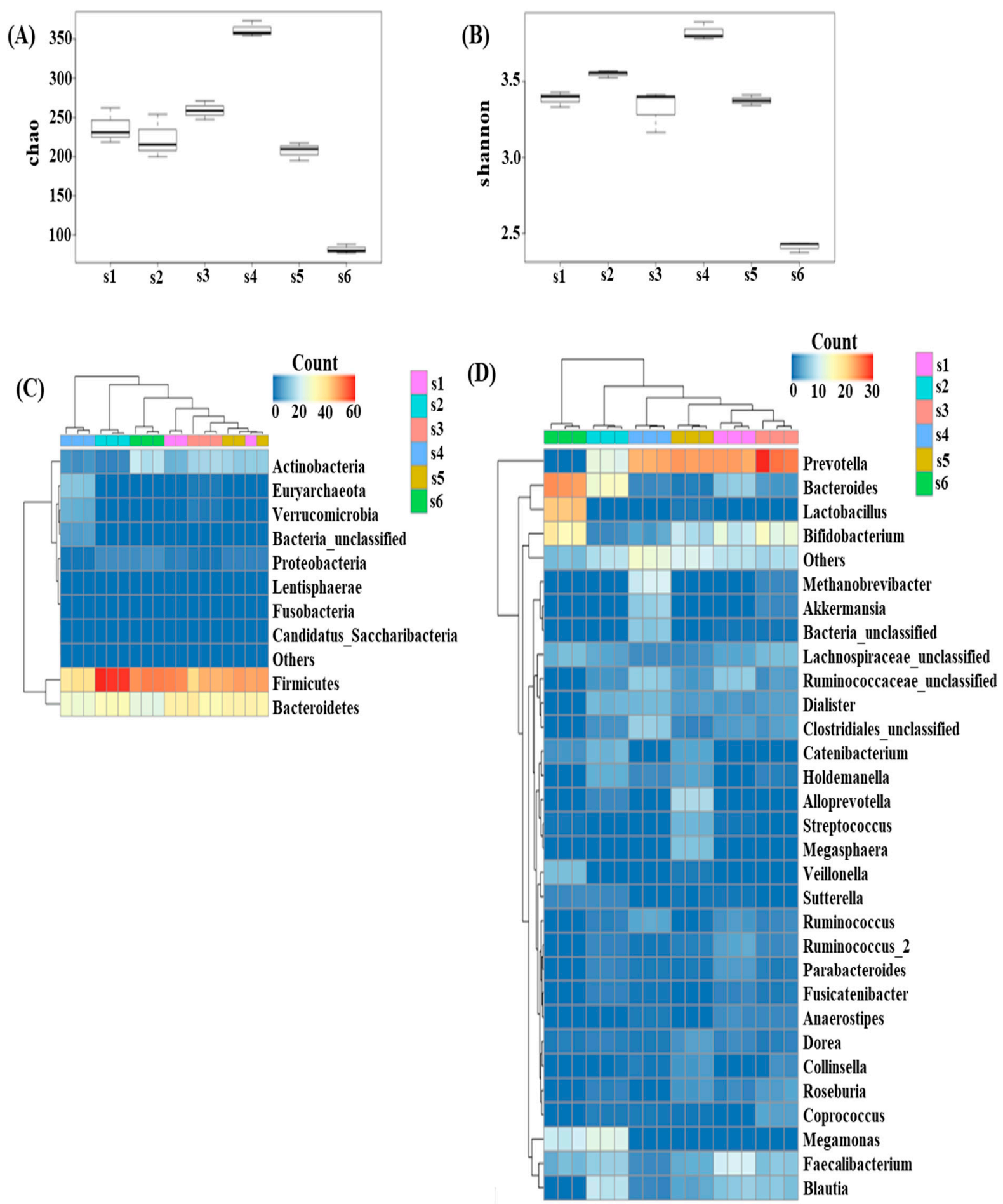

Figure 1. Microbial diversity among the donors (alpha diversity) showed varied richness (A) and diversity (B). Microbial strata among the fecal donors also found to be individualistic at the phylum (C) and genus level (D). 
S1

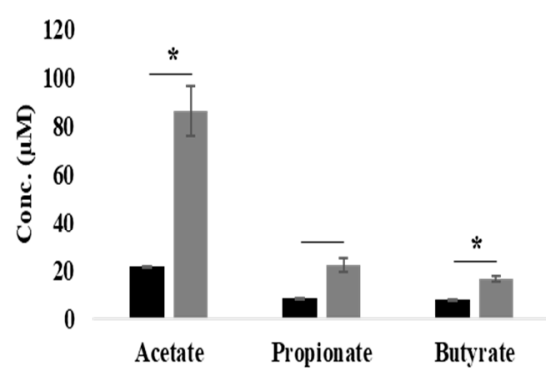

S4

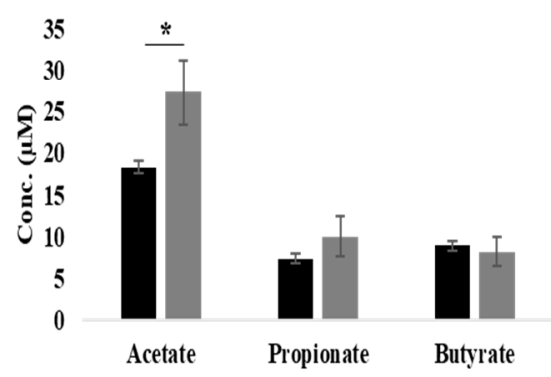

S2

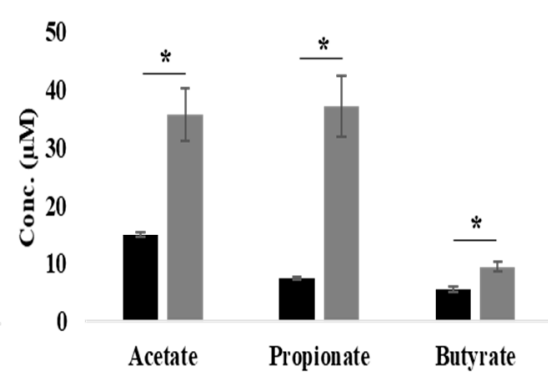

S5

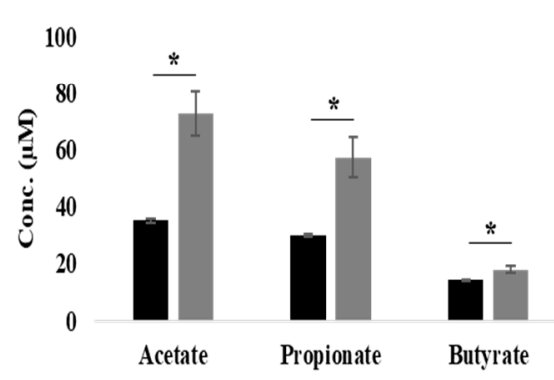

S3

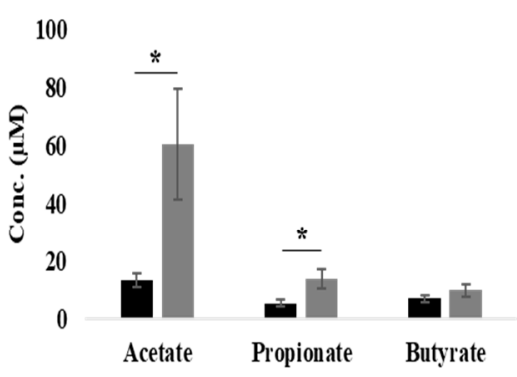

S6

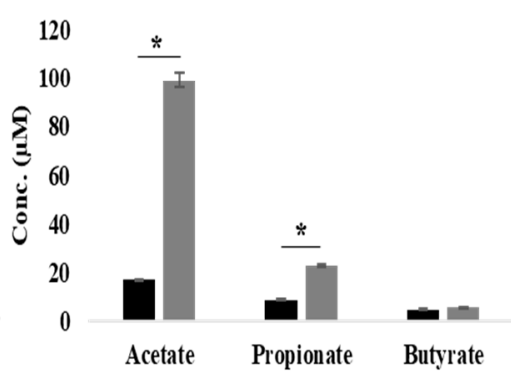

Figure 2. Quantitative comparison of SCFA production in each subject with and without digested beef treatment, as represented by grey and black bars, respectively ( $\left.{ }^{*} ; p<0.05\right)$.

We observed a slight shift in the composition of the individual microbial communities in response to fecal fermentation in the presence of digested beef powder (Figure S3), although none of these shifts appeared to be significant (AMOVA, $p>0.05$ ).

Fecal fermentation induced minor shifts in taxonomic composition at the phylum level. The results shown in Figure 3 show that digested beef powder increased the abundance of both the Firmicutes and Actinobacteria while decreasing the abundance of Bacteroidetes. At the genus level, we observed a decrease in Prevotella and an increase in Bifidobacterium among all subjects except for subject 6, who did not have any Prevotella at initiation. The genus-level composition comparison analysis showed varying degrees of abundance shifts in each subject.

Differential abundance analysis between samples with and without digested beef powder showed that five genera (Bifidobacterium, Blautia, Ruminococcus, Fusicatenibacter, and Holdemanella) were significantly increased in more than four subjects, while another five genera (Bacteroides, Faecalibacterium, Prevotella, Parabacteroides, and Sutterella) were significantly decreased in more than four subjects (Figure 4). In addition, many differentially abundant genera were not common among the subjects. Each subject showed approximately 10-20 increasing genera and 5-10 decreasing genera, likely in response to the digested beef powder. 
(A)

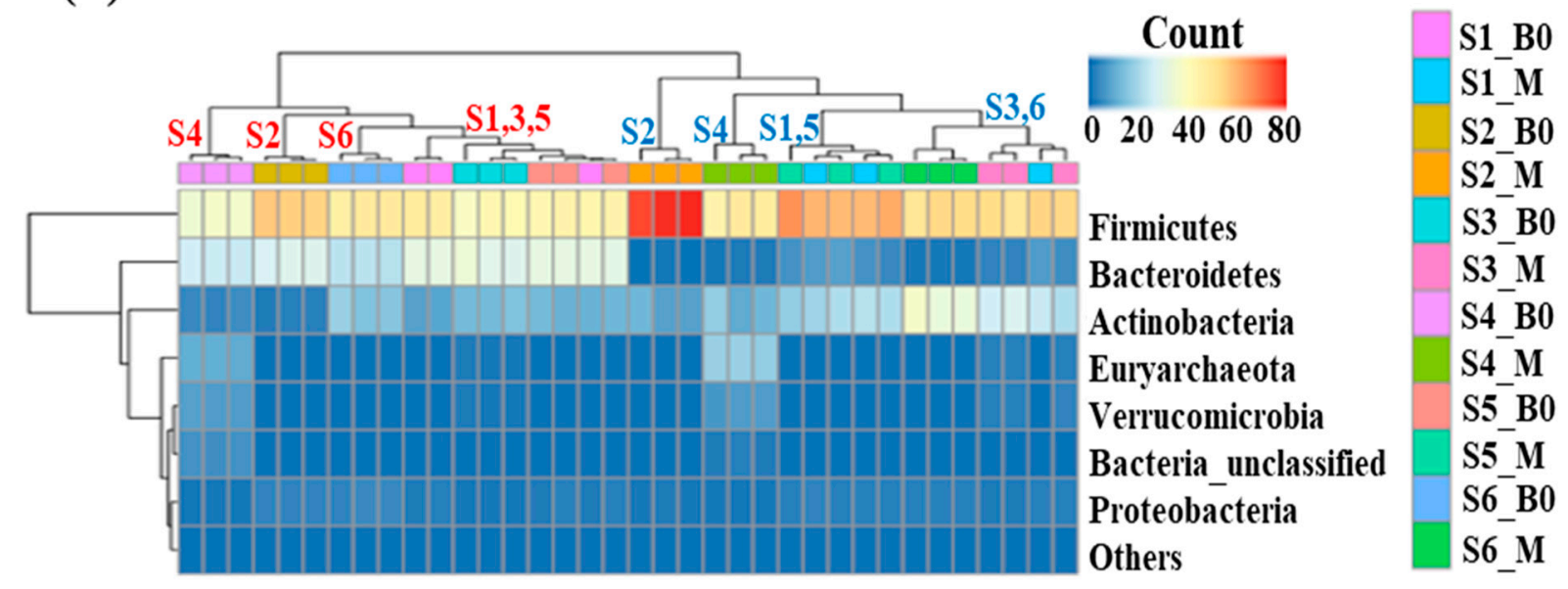

(B)

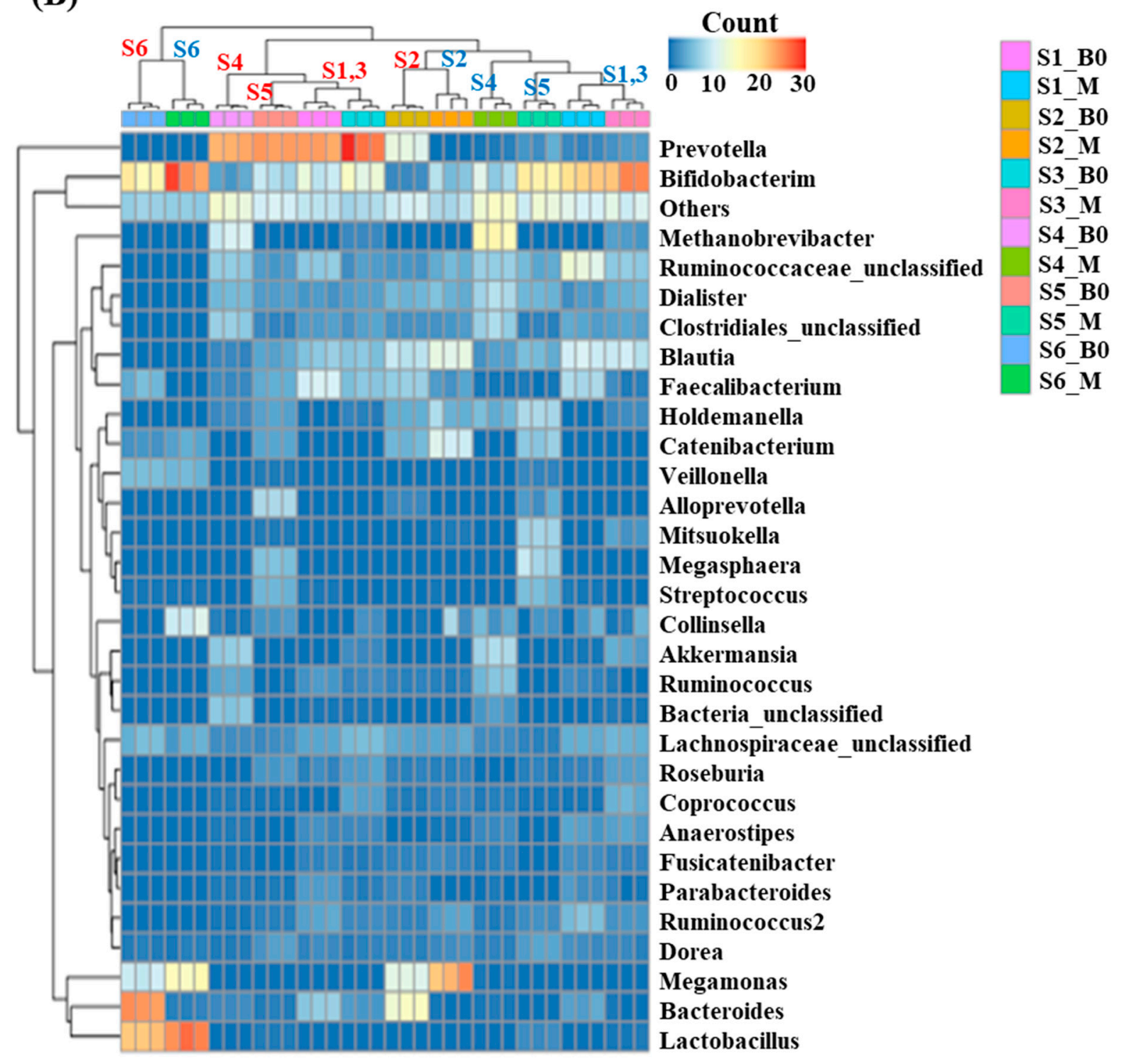

Figure 3. Beef-induced shift in microbial taxonomic composition at the phylum (A) and genus level (B), where red and blue letters indicate subjects with and without the beef treatment, respectively. 
(A)

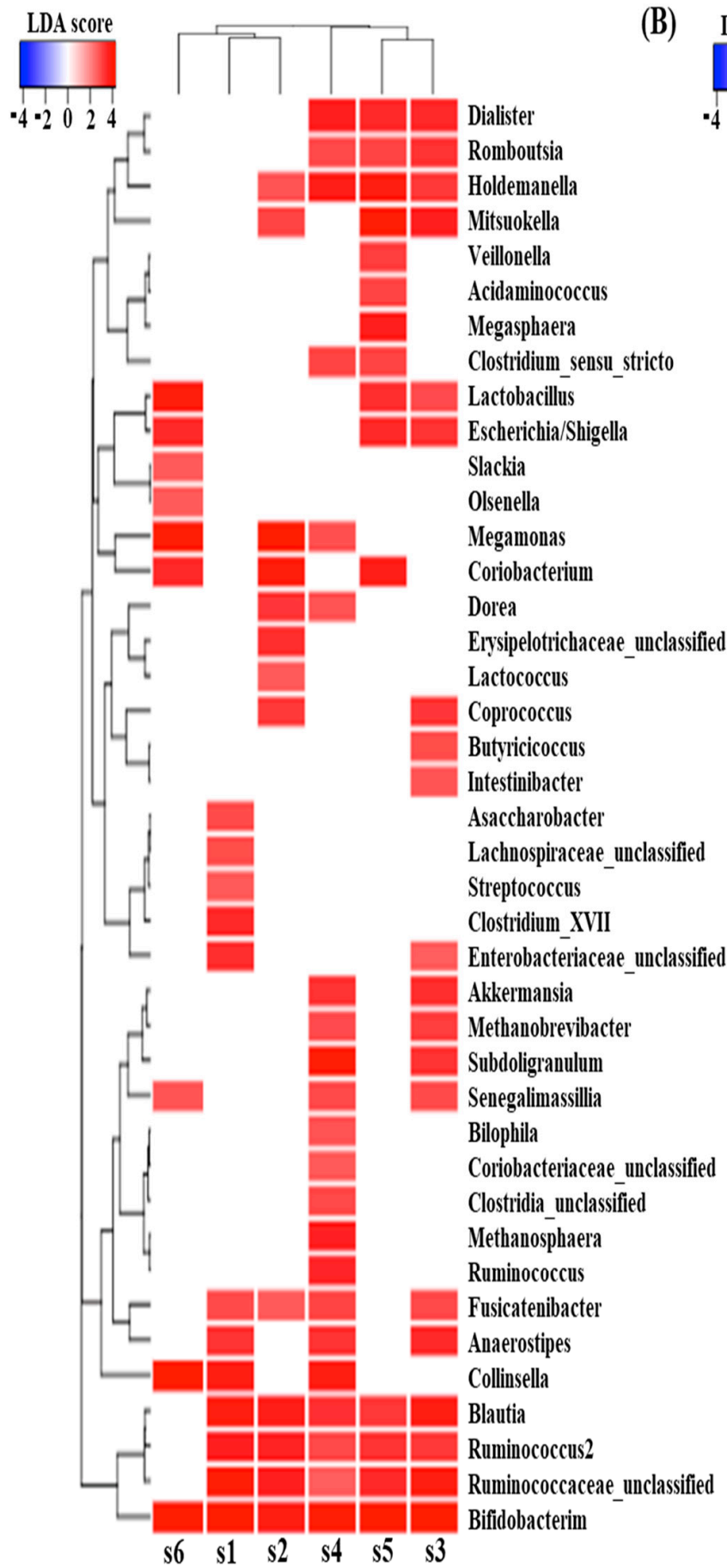

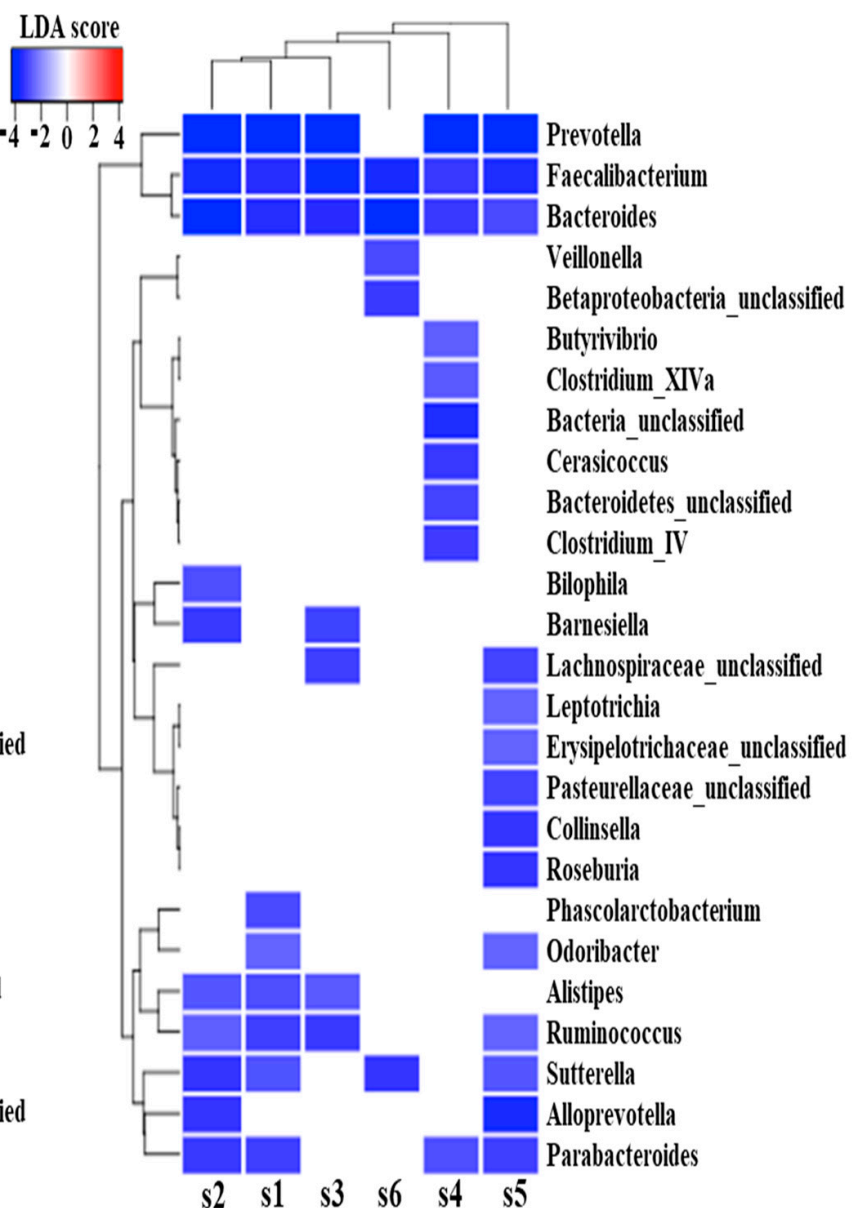

Figure 4. LefSe analysis predicted significantly increased genera (A) and decreased genera (B), following 90 min of fecal fermentation with digested beef.

\subsection{Effect of Digested Beef on Metabolism}

The results in Figure 5 show that digested beef treatment affected the predicted metabolic activity profiles to varying extents in each subject. Figure 5A shows that metabolic activity profiles were shifted in the same direction following fecal fermentation with digested beef powder. On the other hand, the number of metabolic activities demonstrating differential expression varied widely among subjects. Only one and four metabolic activities were affected for subject 1 and 3, respectively, while subjects 2 and 6 demonstrated nearly 50-60 implicated metabolic pathways. 


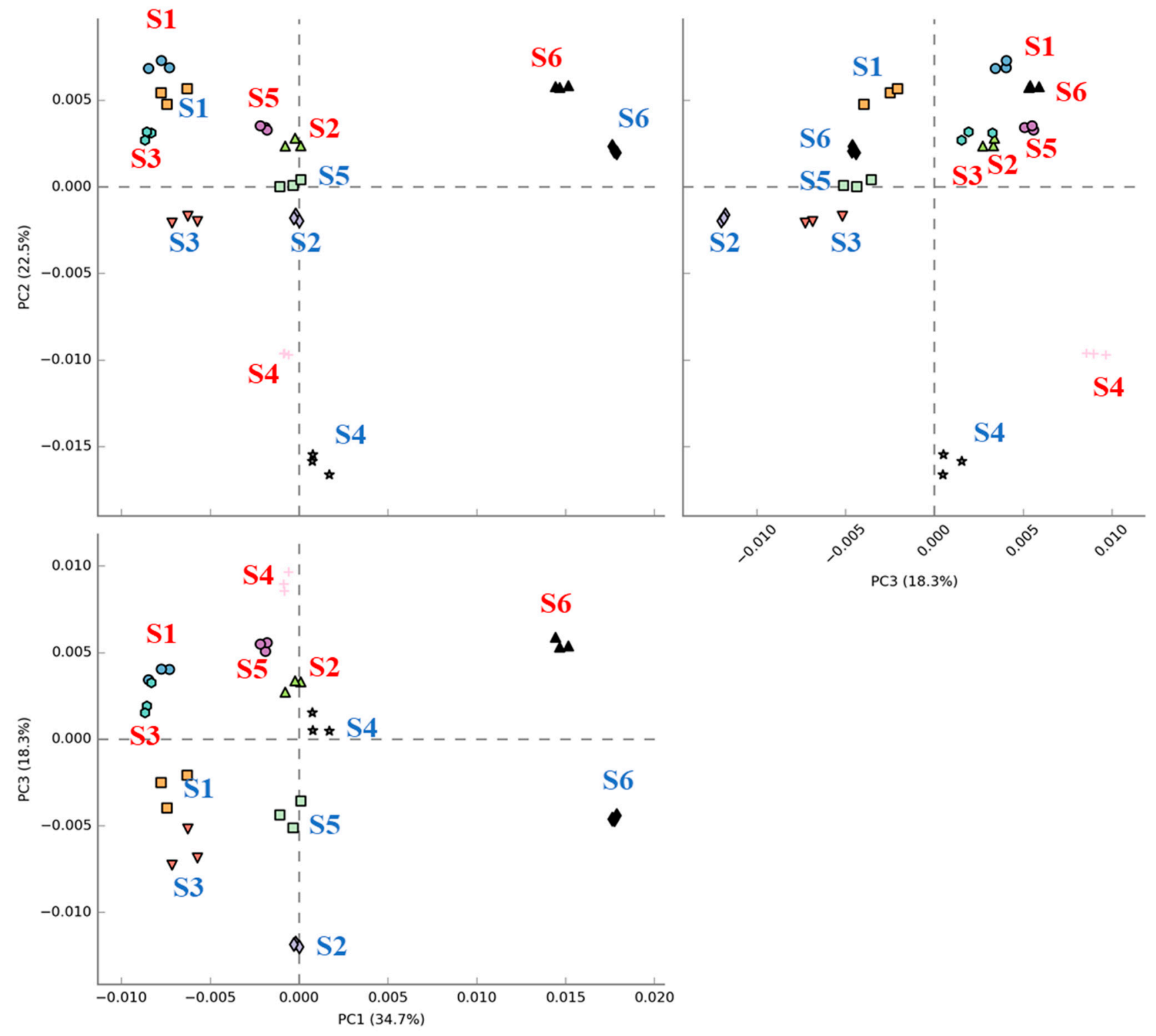

(A)

Figure 5. Cont. 
(B)

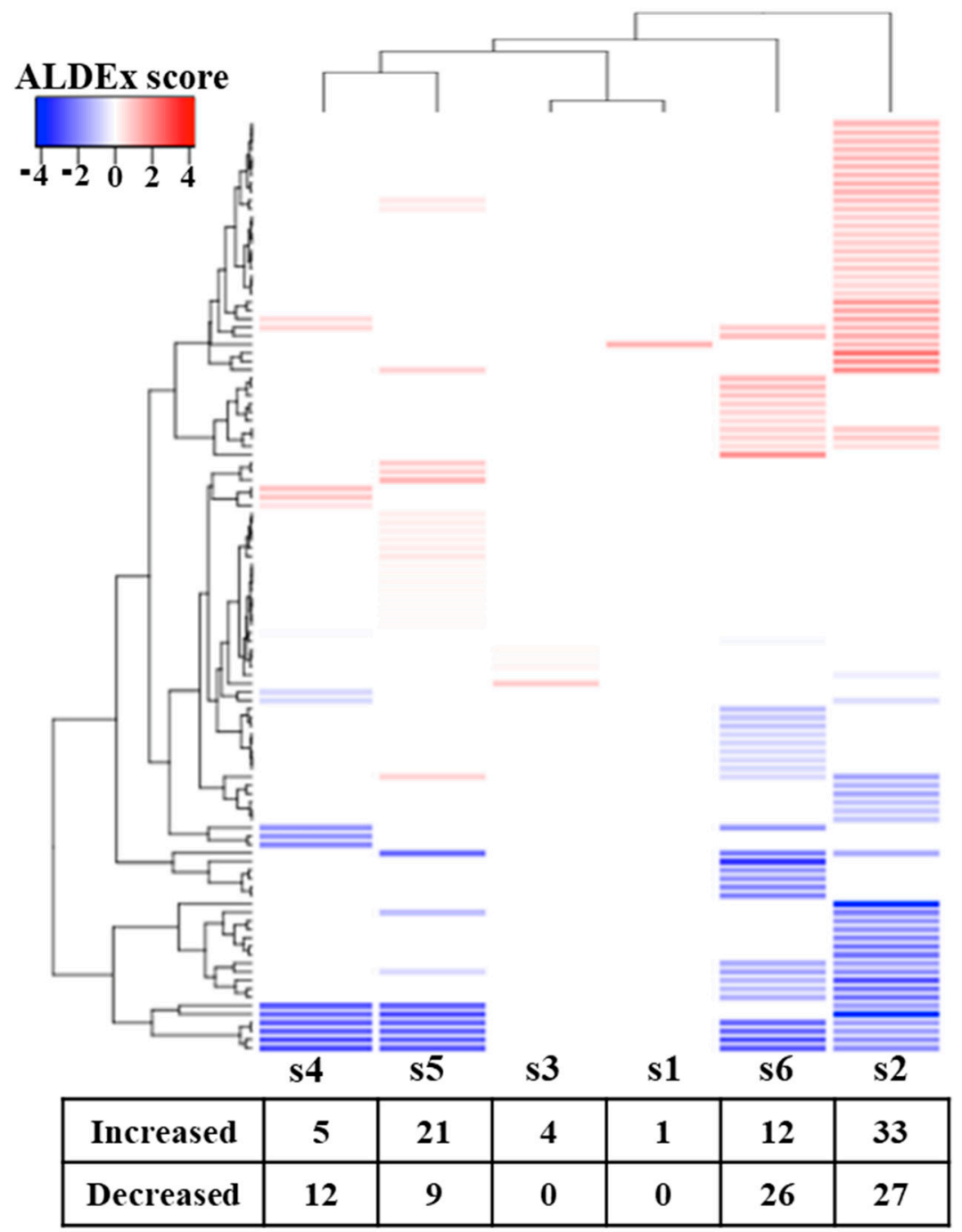

Figure 5. Comparison of PICRUSt-predicted metabolic activities before and after fecal fermentation with digested beef. Principal component analysis (PCA) with red and blue letters indicating subjects before and after fecal fermentation (A). The number of differentially abundant metabolic activities after the addition of the digested beef powder (B).

\section{Discussion}

Our results show that each donor manifested different fecal microbial community types according to the Dirichlet multinomial mixtures model, which implies individual discrepancies in their ability to metabolize specific foods. We also observed different levels of SCFA production in each fecal fermentation, suggesting that individual differences in the microbiota affect fecal microbial metabolic activities. In this study, a significant amount of SCFAs were produced following $90 \mathrm{~min}$ of fecal fermentation in the presence of digested beef powder $(p<0.05)$, with specific variations in each SCFA (Figure S2). In addition, the microbiome was shown to be relatively stable during these $90 \mathrm{~min}$ fermentations (AMOVA, $p>0.05)$, although 5 to 12 genera showed significant differential abundance in each subject (Figure S1). These results suggest that $90 \mathrm{~min}$ of fecal fermentation could be used to investigate how individual microbiomes metabolize digested beef while minimizing the environmental effects of the in vitro incubation. However, the incubation time may vary depending on the food materials.

Given the fact that the gut microbiota plays an important role in digesting food and providing nutrients to hosts, differences in these microbiomes often result in varying effects of diets. Unlike animal models, this study used human microbiomes to investigate the effects of dietary intervention. We observed that fecal fermentation using beef as the carbon 
source produced SCFAs in most of the subjects and increased the abundance of butyrateproducing bacteria such as Bifidobacterium, Ruminococcus, and Blautia. Digested beef has been reported to act as an important protein and nutrient source for Bifidobacterium [2,21,22] and Ruminococcus [23]. Zhu et al. [24] reported that meat proteins increase the abundance of Lactobacillus and the amount of SCFAs produced in the gut of rats, highlighting the types of differences associated with evaluations in human or rat models.

On the other hand, we did not observe a significant increase in butyric acid in half of the subjects, which could be due to a significant decrease in the abundance of Faecalibacterium $(p<0.05)$, a major butyrate producer in the gut, which requires complex carbohydrates for butyrate generation [25]. In addition, the abundance of Prevotella decreased in all subjects except for subject 6, who did not have any Prevotella even at initiation. Faecalibacterium and Prevotella have been reported to be positively associated with complex carbohydrate degradation $[25,26]$. We observed that nearly all the subjects showed significantly reduced numbers of both Prevotella and Bacteroides after 90 min of fecal fermentation using the beef powder as the substrate, which could be due to the lack of fermentable dietary fiber (carbohydrate) necessary to support their growth [27].

Metabolic activity prediction analysis showed that fecal fermentation of beef powders shifted metabolic activity profiles in the same direction (Figure 5A), while differential abundance analysis indicated varying numbers of changes in the metabolic activity of each subject. While there were not many commonly enriched metabolic activities, several metabolic activities were depleted in more than three subjects (Table 1). These depleted metabolic activities were largely linked to long-chain fatty acid biosynthesis, such as oleate, palmitoleate, and stearate, which are known to play protective roles against heart disease. For example, palmitoleate and oleate are monounsaturated fatty acids and are known to reduce the risk of coronary artery disease [28,29]. Unlike other saturated fatty acids, stearate decreases acylcarnitine, a clinical biomarker for the $\beta$-oxidation of fatty acids, and its reduction is associated with improved health in the cardiovascular system [30]. Depletion of this biosynthesis may increase the risk of cardiovascular disease, which is in line with the risks associated with excessive beef consumption reported in other studies [31].

Table 1. Changes in metabolic activities in response to differences in the microbiome of at least 3 subjects.

\begin{tabular}{ccc}
\hline Metabolic Pathways (MetaCyc) & I/D & Description \\
\hline PWY-7664 & $\mathrm{D}$ & Oleate biosynthesis IV (anaerobic) \\
PWYG-321 & $\mathrm{D}$ & Mycolate biosynthesis \\
PWY-6282 & $\mathrm{D}$ & Palmitoleate biosynthesis I (from (5z)-dodec-5-enoate) \\
PWY-5989 & $\mathrm{D}$ & Stearate biosynthesis II (bacteria and plants) \\
FASYN-ELONG-PWY & $\mathrm{D}$ & Fatty acid elongation- saturated \\
PWY0-862 & $\mathrm{D}$ & (5z)-dodec-5-enoate biosynthesis \\
FASYN-INITIAL-PWY & $\mathrm{D}$ & Super pathway of fatty acid biosynthesis initiation (E. Coli) \\
RHAMCAT-PWY & $\mathrm{D}$ & L-rhamnose degradation I \\
PWY-6163 & $\mathrm{I}$ & Chorismite biosynthesis from 3-dehydroquinate \\
\hline
\end{tabular}

${ }^{*}$ I and D indicate increases and decreases, respectively.

In vivo models are labor-intensive and costly and also require ethical approval [32]. Most importantly, differences in individual microbiota are not considered in many of the currently used in vivo models. For these reasons, in vitro digestion models are becoming more commonly used for studying the effects of different dietary substances. While dynamic in vitro models allow the simulation of physiological functions, such as varying $\mathrm{pH}$ and protein substrates, access to such devices is often limited and expensive [33]. In contrast, static in vitro digestion models are rapid and cost-effective and have no ethical issues related to animal handling. 


\section{Conclusions}

Evaluations of in vitro fecal fermentation of beef revealed changes in the microbial community, the predicted metabolic activity profiles of the samples, and the level of microbial metabolites (i.e., SCFAs). Many clinical and preclinical evaluations of dietary intervention assume that the effects seen in animals or in specific groups of people can be universally translated and applied to other people. Our study demonstrates that the effects of a single food can vary depending on the microbiota. Therefore, current dietary intervention evaluation systems should be interpreted with caution. Although further studies on larger populations are required for validation, this study presents a simple and cost-effective protocol for investigating the associations between diet and the personal gut microbiome.

Supplementary Materials: The following are available online at https: / www.mdpi.com/article / 10.3390/app11156841/s1, Figure S1: Differentially abundant genera after 90 min (B90) of in vitro fecal fermentation compared to B0, Figure S2: Quantitative comparison of SCFA production with and without the addition of digested beef powder, for different time point (30,60 and $90 \mathrm{~min})$. Where fecal fermentation of $90 \mathrm{~min}$ beef treated were showing higher level of SCFAs for acetate (A), propionate (B), and butyrate $(\mathrm{C})$. (Dotted line = Beef treatment, continuous line = without beef), Figure S3: The shift in microbial diversity (beta-diversity) between the blank (B) and beef treated (M) samples of six subjects, as visualized using non-metric multidimensional scaling, Table S1: Output from the get.communitytype mothur subroutine. The minimum number of Laplace corresponds to the number of the partition in column ' $\mathrm{K}$ '.

Author Contributions: V.S. performed the experiments, analyzed the data, and drafted the manuscript. Y.-C.R. supervised the work. T.U. designed the study and proofread the manuscript. All authors have read and agreed to the published version of the manuscript.

Funding: This work was supported by the Korea Institute of Planning and Evaluation for Technology in Food, Agriculture, and Forestry (IPET) via the Agricultural Microbiome R\&D Program, funded by the Ministry of Agriculture, Food and Rural Affairs (MAFRA) (918021-4).

Institutional Review Board Statement: Not applicable.

Informed Consent Statement: Not applicable.

Data Availability Statement: The data have been submitted to the SRA repository with BioProject no. PRJNA740098.

Acknowledgments: We acknowledge the Subtropical Agriculture Research Institute (SARI), Jeju National University, South Korea for providing research facility.

Conflicts of Interest: The authors have no conflict of interest to declare.

\section{References}

1. Lepage, P.; Leclerc, M.C.; Joossens, M.; Mondot, S.; Blottière, H.M.; Raes, J.; Ehrlich, D.; Doré, J. A metagenomic insight into our gut's microbiome. Gut 2013, 62, 146-158. [CrossRef] [PubMed]

2. $\quad$ Singh, R.K.; Chang, H.-W.; Yan, D.; Lee, K.M.; Ucmak, D.; Wong, K.; Abrouk, M.; Farahnik, B.; Nakamura, M.; Zhu, T.H.; et al. Influence of diet on the gut microbiome and implications for human health. J. Transl. Med. 2017, 15, 1-17. [CrossRef]

3. De Filippis, F.; Pellegrini, N.; Vannini, L.; Jeffery, I.; La Storia, A.; Laghi, L.; Serrazanetti, D.I.; Di Cagno, R.; Ferrocino, I.; Lazzi, C.; et al. High-level adherence to a Mediterranean diet beneficially impacts the gut microbiota and associated metabolome. Gut 2015, 65, 1812-1821. [CrossRef] [PubMed]

4. Tomova, A.; Bukovsky, I.; Rembert, E.; Yonas, W.; Alwarith, J.; Barnard, N.D.; Kahleova, H. The Effects of Vegetarian and Vegan Diets on Gut Microbiota. Front. Nutr. 2019, 6, 47. [CrossRef]

5. Oliphant, K.; Allen-Vercoe, E. Macronutrient metabolism by the human gut microbiome: Major fermentation by-products and their impact on host health. Microbiome 2019, 7, 1-15. [CrossRef]

6. Scott, K.P.; Gratz, S.W.; Sheridan, P.O.; Flint, H.J.; Duncan, S.H. The influence of diet on the gut microbiota. Pharmacol. Res. 2013, 69, 52-60. [CrossRef]

7. Yang, J.; Yu, J. The association of diet, gut microbiota and colorectal cancer: What we eat may imply what we get. Protein Cell 2018, 9, 474-487. [CrossRef] [PubMed]

8. Brodkorb, A.; Egger, C.; Alminger, M.; Alvito, P.; Assunção, R.; Ballance, S.; Bohn, T.; Bourlieu-Lacanal, C.; Boutrou, R.; Carrière, F.; et al. INFOGEST static in vitro simulation of gastrointestinal food digestion. Nat. Protoc. 2019, 14, 991-1014. [CrossRef] 
9. Minekus, M.; Alminger, M.; Alvito, P.; Ballance, S.; Bohn, T.; Bourlieu, C.; Carrière, F.; Boutrou, R.; Corredig, M.; Dupont, D.; et al. A standardised static in vitro digestion method suitable for food-An international consensus. Food Funct. 2014, 5, 1113-1124. [CrossRef]

10. Peyron, M.-A.; Mishellany, A.; Woda, A. Particle Size Distribution of Food Boluses after Mastication of Six Natural Foods. J. Dent. Res. 2004, 83, 578-582. [CrossRef]

11. Jalabert-Malbos, M.-L.; Mishellany-Dutour, A.; Woda, A.; Peyron, M.-A. Particle size distribution in the food bolus after mastication of natural foods. Food Qual. Prefer. 2007, 18, 803-812. [CrossRef]

12. Moon, J.S.; Li, L.; Bang, J.; Han, N.S. Application of in vitro gut fermentation models to food components: A review. Food Sci. Biotechnol. 2016, 25, 1-7. [CrossRef] [PubMed]

13. Schloss, P.D.; Westcott, S.L.; Ryabin, T.; Hall, J.R.; Hartmann, M.; Hollister, E.B.; Lesniewski, R.A.; Oakley, B.B.; Parks, D.H.; Robinson, C.J.; et al. Introducing mothur: Open-Source, Platform-Independent, Community-Supported Software for Describing and Comparing Microbial Communities. Appl. Environ. Microbiol. 2009, 75, 7537-7541. [CrossRef]

14. Quast, C.; Pruesse, E.; Yilmaz, P.; Gerken, J.; Schweer, T.; Yarza, P.; Peplies, J.; Glöckner, F.O. The SILVA ribosomal RNA gene database project: Improved data processing and web-based tools. Nucleic Acids Res. 2013, 41, D590-D596. [CrossRef]

15. Cole, J.R.; Wang, Q.; Fish, J.A.; Chai, B.; McGarrell, D.M.; Sun, Y.; Brown, C.T.; Porras-Alfaro, A.; Kuske, C.R.; Tiedje, J.M. Ribosomal Database Project: Data and tools for high throughput rRNA analysis. Nucleic Acids Res. 2013, 42, D633-D642. [CrossRef]

16. Clarke, K.R.; Somerfield, P.J.; Chapman, M.G. On resemblance measures for ecological studies, including taxonomic dissimilarities and a zero-adjusted Bray-Curtis coefficient for denuded assemblages. J. Exp. Mar. Biol. Ecol. 2006, 330, 55-80. [CrossRef]

17. Douglas, G.M.; Maffei, V.J.; Zaneveld, J.; Yurgel, S.N.; Brown, J.R.; Taylor, C.M.; Huttenhower, C.; Langille, M.G.I. PICRUSt2: An improved and extensible approach for metagenome inference. bioRxiv 2019, 672295. [CrossRef]

18. Segata, N.; Izard, J.; Waldron, L.; Gevers, D.; Miropolsky, L.; Garrett, W.S.; Huttenhower, C. Metagenomic biomarker discovery and explanation. Genome Biol. 2011, 12, R60. [CrossRef] [PubMed]

19. Holmes, I.; Harris, K.; Quince, C. Dirichlet Multinomial Mixtures: Generative Models for Microbial Metagenomics. PLoS ONE 2012, 7, e30126. [CrossRef] [PubMed]

20. Parks, D.H.; Tyson, G.; Hugenholtz, P.; Beiko, R.G. STAMP: Statistical analysis of taxonomic and functional profiles. Bioinformatics 2014, 30, 3123-3124. [CrossRef]

21. Shen, Q.; Chen, Y.A.; Tuohy, K.M. A comparative in vitro investigation into the effects of cooked meats on the human faecal microbiota. Anaerobe 2010, 16, 572-577. [CrossRef]

22. Reddy, B.S.; Weisburger, J.H.; Wynder, E.L. Effects of High Risk and Low Risk Diets for Colon Carcinogenesis on Fecal Microflora and Steroids in Man. J. Nutr. 1975, 105, 878-884. [CrossRef]

23. Biddle, A.; Stewart, L.; Blanchard, J.L.; Leschine, S. Untangling the Genetic Basis of Fibrolytic Specialization by Lachnospiraceae and Ruminococcaceae in Diverse Gut Communities. Diversity 2013, 5, 627-640. [CrossRef]

24. Albracht-Schulte, K.; Islam, T.; Johnson, P.; Moustaid-Moussa, N. Systematic Review of Beef Protein Effects on Gut Microbiota: Implications for Health. Adv. Nutr. 2020, 12, 102-114. [CrossRef]

25. Adamberg, K.; Tomson, K.; Talve, T.; Pudova, K.; Puurand, M.; Visnapuu, T.; Alamäe, T.; Adamberg, S. Levan Enhances Associated Growth of Bacteroides, Escherichia, Streptococcus and Faecalibacterium in Fecal Microbiota. PLoS ONE 2015, 10, e0144042. [CrossRef] [PubMed]

26. Sandberg, J.; Kovatcheva-Datchary, P.; Björck, I.; Bäckhed, F.; Nilsson, A. Abundance of gut Prevotella at baseline and metabolic response to barley prebiotics. Eur. J. Nutr. 2018, 58, 2365-2376. [CrossRef] [PubMed]

27. Chen, T.; Long, W.; Zhang, C.; Liu, S.; Zhao, L.; Hamaker, B.R. Fiber-utilizing capacity varies in Prevotella- versus Bacteroidesdominated gut microbiota. Sci. Rep. 2017, 7, 1-7. [CrossRef]

28. Parveez, G.K.A.; Rasid, O.A.; Hashim, A.T.; Ishak, Z.; Rosli, S.K.; Sambanthamurthi, R. 4-Tissue Culture and Genetic Engineering of Oil Palm. In Palm Oil; Lai, O.-M., Tan, C.-P., Akoh, C.C., Eds.; AOCS Press: Champaign, IL, USA, 2012; pp. 87-135.

29. Lopez, S.; Bermudez, B.; la Paz, S.M.; Pacheco, Y.M.; Ortega-Gomez, A.; Varela, L.M.; Lemus-Conejo, A.; Millan-Linares, M.C.; Rosillo, M.A.; Abia, R.; et al. Oleic Acid: The Main Component of Olive Oil on Postprandial Metabolic Processes. In Olives and Olive Oil in Health and Disease Prevention; Preedy, V.R., Watson, R.R., Eds.; Academic Press: San Diego, CA, USA, 2010; Chapter 154; pp. 1385-1393.

30. Senyilmaz-Tiebe, D.; Pfaff, D.H.; Virtue, S.; Schwarz, K.V.; Fleming, T.; Altamura, S.; Muckenthaler, M.U.; Okun, J.G.; Vidal-Puig, A.; Nawroth, P.; et al. Dietary stearic acid regulates mitochondria in vivo in humans. Nat. Commun. 2018, 9, 1-10. [CrossRef] [PubMed]

31. Mota, J.S.D.O.; Boué, G.; Guillou, S.; Pierre, F.; Membré, J.-M. Estimation of the burden of disease attributable to red meat consumption in France: Influence on colorectal cancer and cardiovascular diseases. Food Chem. Toxicol. 2019, 130, 174-186. [CrossRef]

32. Bohn, T.; Carriere, F.; Day, L.; Deglaire, A.; Egger, L.; Freitas, D.; Golding, M.; le Feunteun, S.; Macierzanka, A.; Menard, O.; et al. Correlation between in vitro and in vivo data on food digestion. What can we predict with static in vitro digestion models? Crit. Rev. Food Sci. Nutr. 2018, 58, 2239-2261. [CrossRef] [PubMed]

33. Mulet-Cabero, A.; Egger, L.; Portmann, R.; Ménard, O.; Marze, S.; Minekus, M.; le Feunteun, S.; Sarkar, A.; Grundy, M.M.-L.; Carrière, F.; et al. A standardised semi-dynamic in vitro digestion method suitable for food-An international consensus. Food Funct. 2020, 11, 1702-1720. [CrossRef] [PubMed] 